\title{
New Onset Diabetes After Transplant
}

National Cancer Institute

\section{Source}

National Cancer Institute. New Onset Diabetes After Transplant. NCI Thesaurus. Code C114852.

Development of diabetes after transplant, usually associated with calcineurin inhibitor use. 